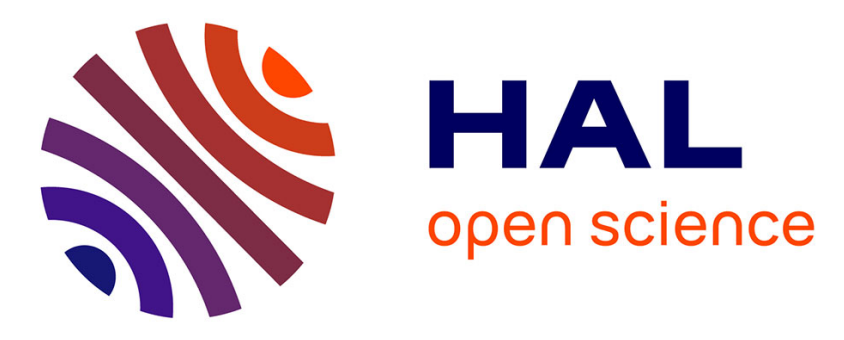

\title{
Anisotropic Time-Resolved Dynamics of Crystal Growth Induced by a Single Laser Pulse Nucleation
}

Sarah Al Gharib, Abdel Karim El Omar, Adnan Naja, Ariane

Deniset-Besseau, Sergey Denisov, Pascal Pernot, Mehran Mostafavi, Jacqueline Belloni

\section{To cite this version:}

Sarah Al Gharib, Abdel Karim El Omar, Adnan Naja, Ariane Deniset-Besseau, Sergey Denisov, et al.. Anisotropic Time-Resolved Dynamics of Crystal Growth Induced by a Single Laser Pulse Nucleation. Crystal Growth \& Design, 2021, 21 (2), pp.799-808. 10.1021/acs.cgd.0c01016 . hal-03322576

\section{HAL Id: hal-03322576 https://hal.science/hal-03322576}

Submitted on 26 Oct 2021

HAL is a multi-disciplinary open access archive for the deposit and dissemination of scientific research documents, whether they are published or not. The documents may come from teaching and research institutions in France or abroad, or from public or private research centers.
L'archive ouverte pluridisciplinaire HAL, est destinée au dépôt et à la diffusion de documents scientifiques de niveau recherche, publiés ou non, émanant des établissements d'enseignement et de recherche français ou étrangers, des laboratoires publics ou privés. 
This document is confidential and is proprietary to the American Chemical Society and its authors. Do not copy or disclose without written permission. If you have received this item in error, notify the sender and delete all copies.

\section{Anisotropic Time-Resolved Dynamics of Crystal Growth Induced by a Single Laser Pulse Nucleation}

\begin{tabular}{|r|l|}
\hline Journal: & Crystal Growth \& Design \\
\hline Manuscript ID & cg-2020-01016j.R1 \\
\hline Manuscript Type: & Article \\
\hline Duthor: & n/a \\
\hline Complete List of Authors: & $\begin{array}{l}\text { Al Gharib, Sarah; Institut de Chimie Physique, Chemistry } \\
\text { EL OMAR, Abdel Karim; Université Libanaise } \\
\text { Naja, Adnan; Université Libanaise } \\
\text { Deniset-Besseau, Ariane; Institut de Chimie Physique } \\
\text { Denisov, Sergey A.; Institut de Chimie Physique } \\
\text { PERNOT, Pascal; CNRS } \\
\text { Mostafavi, Mehran; Universite Paris-Saclay, Institute de chimie physique } \\
\text { Belloni, Jacqueline; Institut de Chimie Physique }\end{array}$ \\
\hline &
\end{tabular}

\section{SCHOLARONE" \\ Manuscripts}




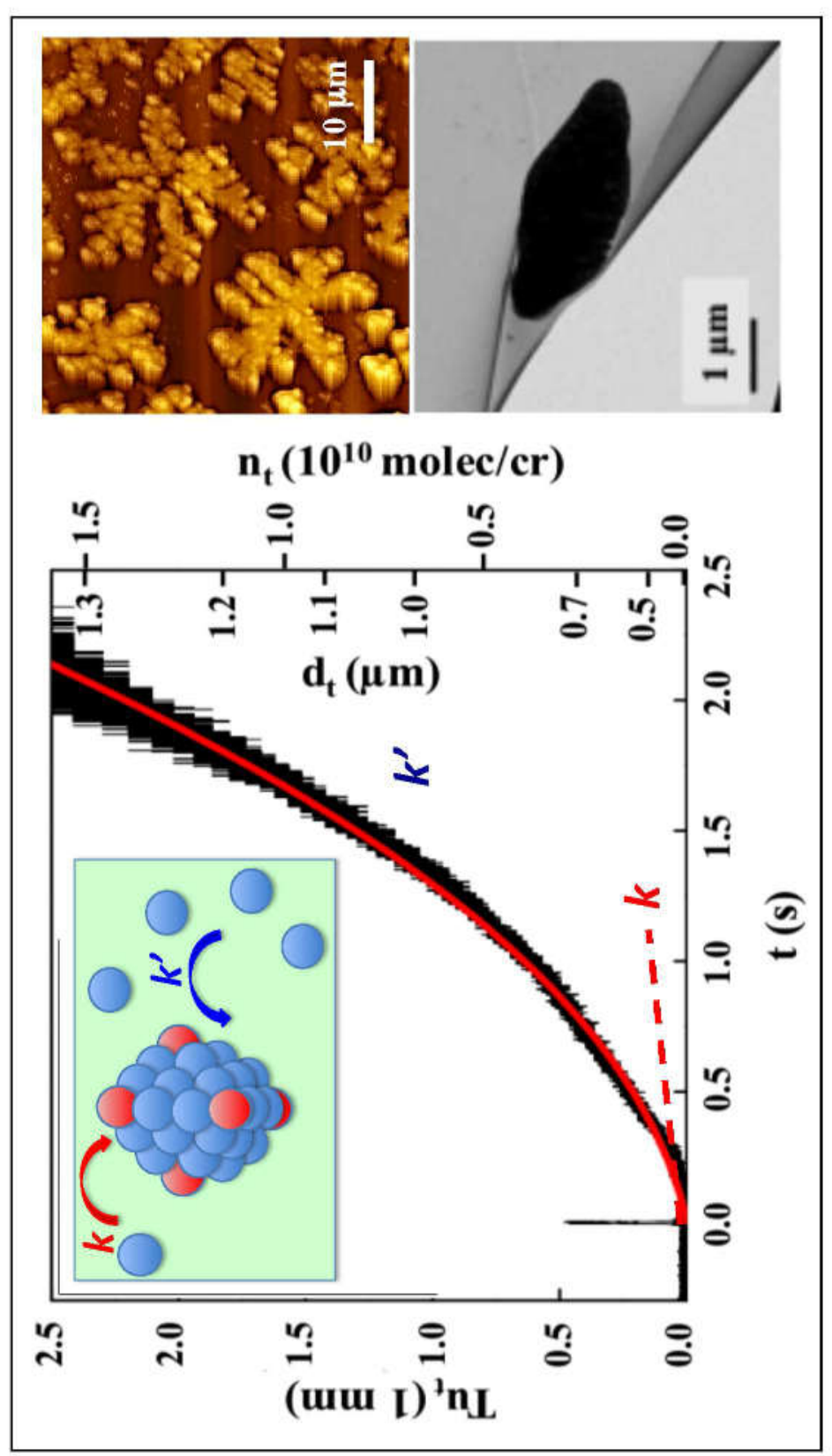

ACS Paragon Plus Environment 


\title{
Anisotropic Time-Resolved Dynamics of Crystal Growth Induced by a Single Laser Pulse Nucleation
}

\author{
Sarah Al Gharib, ${ }^{1,2}$ Abdel Karim El Omar, ${ }^{2}$ Adnan Naja, ${ }^{2}$ Ariane Deniset-Besseau, ${ }^{1}$ Sergey A. \\ Denisov, ${ }^{1}$ Pascal Pernot, ${ }^{1}$ Mehran Mostafavi, ${ }^{1}$ Jacqueline Belloni ${ }^{1}$
}

Université Paris-Saclay, CNRS, Institut de Chimie Physique, 91405 Orsay, France. Université Libanaise, Laboratoire Physique et Modélisation, 1300 Tripoli, Lebanon

\begin{abstract}
Nucleation and growth of crystallites are induced by the shockwave of a single laser nanosecond pulse in highly supersaturated solutions of potassium nitrate (saltpeter). The kinetics of the turbidity resulting from the light scattering by the growing crystals is observed by time-resolved optical spectroscopy. With an optical path of $10 \mathrm{~mm}$, we first focused on the early part of the growth under various conditions, but it is limited by the detection system to a few percent of the total growth. Remarkably, in this domain and for all supersaturated concentrations studied, the turbidity increases proportionally to time. This zero-order process with an accretion rate constant $\left(k=4.7 \times 10^{7} \mathrm{~L} \mathrm{~mol}^{-1} \mathrm{~s}^{-1}\right)$ implies that the nuclei and growing crystals concentration remains constant. This concentration is determined from the final diameter of crystals that are seen of bipyramidal shape by TEM. Moreover, the number of accretion sites per crystal must be constant in this time range and they are identified as the apexes. In complement, the kinetics detected with shorter optical paths permits to observe larger parts of the total growth (up to $35 \%$ with $1 \mathrm{~mm}$ of optical path). In this case, after the linear part, the turbidity increase is markedly accelerated, indicating that the number of accretion sites per crystal increases rapidly with the crystal size and that the edges and facets sites are also fixing the molecules. The complete analysis by a homogeneous kinetics model of the signals obtained under various conditions concludes to an anisotropic growth of the crystals with another accretion rate constant $\left(k^{\prime}=100 \mathrm{~L} \mathrm{~mol}^{-1} \mathrm{~s}^{-1}\right)$ much lower on these edges and facets sites than on the apexes. However, this contribution becomes predominant only when the crystals develop to a larger size.
\end{abstract}

\section{INTRODUCTION}

The knowledge of nucleation/crystallization processes is crucial in many natural domains, such as climatology, geology, mineralogy, biomineralization, and in industry, such as metallurgy engineering, large monocrystals synthesis or pharmaceutics. A great number of experimental data have been obtained on the crystallization of pure materials ${ }^{1}$ by creating nuclei in supersaturated solutions after cooling the solutions (when the solubility increases with the temperature), evaporating the solvent or adding an antisolvent. A chemical precipitation may be also used after mixing by microfluidics. ${ }^{1}$ However, these methods are often slow in creating the new phase compared to the crystal growth rate. Therefore, the nucleation occurs along this evolution of the conditions and strongly depends on them. The growth kinetics is hence usually observed at long time only.

In contrast, a sudden nucleation may be induced by the shock-wave delivered by train or single laser pulses in metastable supersaturated solutions transparent to the laser wavelength (non-photochemical laser induced nucleation or NPLIN).2,3,4,5,6,7,8,9 Various types of crystals have been induced recently by femtosecond laser pulses focused in supersaturated solutions. ${ }^{10,11,12,13,14,15}$ The rate of crystallization was derived theoretically. ${ }^{16}$ Single crystals have been induced by infrared laser pulses locally heating glass samples. ${ }^{16,17,18}$

The final size and structure of the nanocrystals depend on the irradiation conditions, especially on the beam polarization. Theoretically, the classical nucleation theory (CNT) of Zeldovich, 19 and more recent developments ${ }^{20,21}$ explain the existence of a metastable supersaturated phase. Crystallogenesis is induced only by supercritical nuclei of size large enough to fix on their surface supplementary molecules coming from the surrounding phase faster than the evaporation/dissolution of the superficial molecules. Kinetically, the probability of encounters in the supersaturated phase implies that the nucleation starts by the formation of the dimer that displays the lowest binding energy. ${ }^{22}$ Thus the 
metastability domain strictly extends between the evaporation/dissolution temperature of macroscopic crystals and the dissociation equilibrium temperature of the dimers into monomers. ${ }^{22}$ The free energy of $\mathrm{CaCO}_{3}$ nanoparticles has been computed, and an initial formation of an amorphous phase before crystallization has been demonstrated. 22 The atomic simulation of the crystallization of liquid $\mathrm{SiO}_{2}$ to $\beta$-cristobalite used the XRD peak intensities as collective variables. ${ }^{23}$ Due to their nonlinear optical properties, the growth kinetics of single large potassium dihydrogeno-phosphate crystals (KDP), induced on germs, was extensively studied under high purity and stationary supersaturation conditions. ${ }^{24,25}$ The results were analyzed by a rapid transport model (RTM). ${ }^{25}$ Also germ-mediated growth kinetics of single millimetric potassium nitrate crystals was investigated and the different rates measured at various supersaturation concentrations and on various crystal faces. ${ }^{26}$ Imaging of the growth features at micrometer range was observed by scanning force microscopy. ${ }^{27}$ The influence of various proteins on the growth rate of calcite crystals has been compared. ${ }^{28}$ The transition dynamics from the amorphous state to the crystalline state and the growth of $\mathrm{YVO}_{4}: \mathrm{Eu}$ produced by microfluidics has been observed by timeresolved small angle scattering of synchrotron X-rays. ${ }^{29}$

Another experimental approach is focused on the early homogeneous kinetics of the growth at submicrometric size range, instead of observing the crystallographic structure of the crystals. The principle of the growth dynamics study of crystals has been already described in detail. 30 For that purpose, much higher local supersaturation concentrations are suddenly induced at room temperature by the very short shockwave of a powerful and focused laser single pulse. The perturbation induces a local concentration increase over the limit of the metastability domain. It has been shown that this limit is thermodynamically imposed by the formation equilibrium of dimers ${ }^{16}$ which then grow rapidly during the system relaxation up to the critical nuclei required for the further spontaneous growth under relaxed conditions. The nuclei growth dynamics is then observed by the time-resolved turbidity in the solution. The induction time of the nuclei within the pulse is thus separated from the further growth time, and both are shorter than any new spontaneous creation of nuclei. The concentration of laser-induced growing nuclei is very small compared to that of the supersaturated molecules and the nuclei coalescence is totally negligible compared to their growth by accretion of supplementary molecules. Note that the shockwave also induces a quasi-instantaneous dispersion of the nuclei even if they were produced in the confined region of the laser focusing (see section 3.2). The kinetics signals of the growth may be analyzed according to the homogeneous kinetics model (HKM) and give access to the absolute rate constants of the growth by accretion of supersaturated molecules on the nuclei. ${ }^{24}$ In this previous study, optical cells with an optical path of $1 \mathrm{~cm}$ were used in order to observe the nucleation and growth of crystallites by the turbidity change as early as possible. It appeared that the time-dependent turbidity increases linearly with time at various supersaturation concentrations. These results supported that the growth is based on an autocatalytic cascade of accretion/dissolution processes on a constant concentration of growing nuclei and then particles. However, the turbidity increased rapidly and reached after a short time the system detection limits. Therefore, the observed dynamics corresponded to only a few percents of the complete growth up to the final size. ${ }^{31}$

The aim of the present work is to overcome this limitation in adapting the experimental device in order to study the same phenomenon in cuvettes of much shorter optical path, then to investigate the growth during a larger part of the time-resolved dynamics and to derive the mechanism by the homogeneous kinetics model.

\section{EXPERIMENTAL SECTION}

\subsection{Materials}

Potassium nitrate $\left(\mathrm{KNO}_{3}\right.$, saltpeter, from Sigma Aldrich) $\left(\mathrm{Mw}=101.1 \mathrm{~g} \mathrm{~mol}^{-1}\right.$, specific mass $\rho=2.109 \mathrm{~g}$ ) is a very soluble salt ( $30 \mathrm{~g} / 100 \mathrm{~g} \mathrm{H}_{2} \mathrm{O}$ at room temperature and normal pressure conditions). Crystals have an orthorhombic structure with three distinct lattice vectors, and for the lattice cell: $a=5.4119 \AA, b=9.1567 \AA$, and $c=$ $6.4213 \AA$. The solubility increases with the temperature. Supersaturated solutions were obtained by heating, in a water bath up to $60{ }^{\circ} \mathrm{C}$ (measured by a thermometer in the bath), $4 \mathrm{~mL}$ of Millipore water (resistivity $18.2 \mathrm{M} \Omega \mathrm{cm}$ ) and a weighed amount (2.2 to $3.0 \mathrm{~g}$ ) of potassium nitrate crystals in a small, tapped and extremely clean glass bottle. $^{2}$ After the salt dissolution, this stock volume increases (up to 5.0 and $5.5 \mathrm{~mL}$, or 125 to $137 \%$, respectively, instead of $110 \%$ at saturation). The final saturated concentration is thus $c_{\text {sat }}=2.7 \mathrm{M}$ and the supersaturated solution is $c_{0}=4.35$ to $5.5 \mathrm{M}$ (saturation index $s=c_{0} / c_{s a t}=1.6$ to 2.05 , or relative saturation index $\sigma=\left(c_{0}-c_{s a t}\right) / c_{s a t}=0.6$ to 1.03$)$. In order to prevent any early nucleation, the adequate volume of solution was gently transferred while hot with a one-use plastic pipette into a one-use polystyrene optical cuvette (VWR). Several types of cuvettes were used with all their faces transparent but with different shapes (Figure S1, Supporting Information). The sizes were $10 \times 10,10 \times 4$ or $10 \times 2 \mathrm{~mm}^{2}$. In addition, we used, as a spacer inside the $10 \times 2 \mathrm{~mm}^{2}$ cuvette, a glass slide $10 \mathrm{~mm}$ wide and $1 \mathrm{~mm}$ thick, and the optical path was only $1 \mathrm{~mm}$. The glass spacer was heated before use and adding the solution. The sample volumes submitted to the laser shockwave were from 0.065 to $1 \mathrm{~mL}$. Then, the solution was let to cool down in the cuvette to $20{ }^{\circ} \mathrm{C}$ in ambient air without vibration. The room temperature was controlled and fixed at $20{ }^{\circ} \mathrm{C}$. Under these conditions, the high surface/volume ratio of the sample assured a homogeneous cooling. The cooling time decreases when the volume of the supersaturated solution decreases. It is only $1 \mathrm{~min}$ for the cuvette with $1 \mathrm{~mm}$ of optical path where the volume is very small. The cuvette is firmly fixed in the sample holder. Then, the laser pulse shock was delivered immediately after the cooling step. So, we minimized the spontaneous nucleation that may arise in supersaturated solutions, especially at increasing time between the cooling time and the laser pulse shock (without a shock, the first crystallites would be visible after $1 \mathrm{~min}$ for $5.5 \mathrm{M}$ or $3 \mathrm{~min}$ for $4.3 \mathrm{M}$ ). Therefore, the concentration of possible spontaneous nuclei is negligible compared to the much higher concentration of laser pulseinduced nuclei. 


\subsection{Laser}

The crystallization was induced using second harmonics (532 nm) of fundamental output at $1064 \mathrm{~nm}$ of $\mathrm{Nd}$ :YAG Quanta-Ray Spectra-Physics laser, providing powerful pulses of $250 \mathrm{~mJ}$ with pulse duration of $7 \mathrm{~ns}$. The laser beam was focused in the center of the sample by a lens of 5 $\mathrm{cm}$ focus. The energy deposited by the laser pulse depends on the focusing. The tight focusing used in ref 31 induced high concentrations of nuclei but was very difficult to reproduce from day to day. In the present study, we preferred to fix the focusing at a lens position where the energy absorbed is lower but more constant, and sufficient for producing a homogeneous suspension of crystallites. The $\mathrm{KNO}_{3}$ supersaturated solutions are transparent within a broad range of wavelength from the absorption band edge of nitrate at $350 \mathrm{~nm}$ to beyond $800 \mathrm{~nm}$, especially at the laser wavelength, and no photochemical nucleation may occur (NPLIN).

The turbidity of nascent crystallites is independent of the detection wavelength. ${ }^{31}$ The analyzing beam delivered by a $630 \mathrm{~nm}$ He-Ne laser was perpendicular to the excitation laser beam. By using a lens and a window, the analyzing beam was adapted to the sample size.

The intensity of the light transmitted through the cuvette was filtered by a monochromator (essentially to eliminate possible $532 \mathrm{~nm}$ reflections) and detected by a photomultiplier. The time-resolved signal was acquired by a digitized oscilloscope (Figure S2). The same trigger was used to synchronize the digitized oscilloscope and the single laser-pulse inducing the sudden nucleation and crystallization in the sample. The phase transition after the pulse was observed via the increase of the turbidity $T u_{t}$ per $\mathrm{cm}$ of the optical path $l$ in the solution $T u_{t}=\ln \left(I_{0} / I_{t}\right)$, where $I_{0}$ and $I_{t}$ are the light intensities before and at a time delay $t$ after the pulse, respectively. The turbidity was found proportional to the concentration of molecules incorporated in the crystals with a molar turbidity coefficient $\gamma_{T u}$ per unit of optical path $l$, equal to $\gamma_{T u}=(30 \pm$ 4) $\mathrm{L} \mathrm{mol}^{-1} \mathrm{~cm}^{-1}{ }^{31}$ Note that, because the kinetics model involves the concentration of molecules included in the crystal at time $t$, this coefficient addresses the concentration of these molecules (see SI for the discussion on another coefficient depending on the particle diameter). The turbidity is thus equal to $T u_{t}=\gamma_{T u} \times I \times n_{t} \times c_{n, t}$, where $n_{t}$ is the number of molecules per crystallite and $c_{n, t}$ the concentration of the crystallites.

\subsection{Imaging}

At the end of the rapid growth, we observe a gel-like slurry containing a high concentration of the small crystallites. After the pulse, in order to prevent the slow Ostwald maturation (i.e. the growth of large crystals at the expenses of small ones), a small amount of the crystal suspension was rapidly deposited on glass slides for AFM characterization and on copper grids for TEM (STEM Jeol 1400 ) imaging. The crystallites were rapidly washed with deionized water in order to remove the saturated solution (which may contribute to a supplementary growth of the crystals during the droplet evaporation), and to separate the crystals as much as possible. Finally, the samples were dried. In some cases, during the drying of the droplets, the crystals attracted each other (see section 3.4).
AFM imaging was performed with a NanoIR1 (Anasys Instruments, CA USA). The tapping mode in air was used (cantilever frequency $=325 \mathrm{kHz}$, stiffness $=40 \mathrm{~N} / \mathrm{m}$ ). At least 3 different regions of each sample were investigated using a scan rate of $0.1 \mathrm{~Hz}$, windows scan $80 \mu \mathrm{m} \times 80 \mu \mathrm{m}$ and 500 points by line.

\section{RESULTS AND DISCUSSION}

In highly supersaturated solutions, the laser shockwave induces rapidly the formation of a gel-like slurry of high concentration of very small crystals. During this range of time, the laser-induced nuclei concentration is therefore much higher than in the spontaneous nucleation which is negligible. During the growth, the distribution of the supersaturated molecules over a great number of nuclei results into small particles that remain in homogeneous suspension up to the final size as shown below by the influence of the solution volume on the kinetics. Thus the time-resolved signals may be analyzed by a homogeneous kinetics model (HKM) and give access to the absolute rate constant of the growth by accretion of supersaturated molecules on the nuclei.

In the present study, we first evaluated the influence of the different parameters, namely the laser energy deposition, the sample volume, the supersaturated concentration and the optical path, in order to deduce the nucleation and growth mechanism.

\subsection{Laser energy influence}

As often reported in the literature, the growth kinetics and the final crystal size are very sensitive to the experimental conditions. Among them, the most important is the energy deposition by the shockwave that generates a nuclei concentration which may vary from pulse to pulse, particularly when the beam is extremely focused. For that reason, the beam was less focused in the present

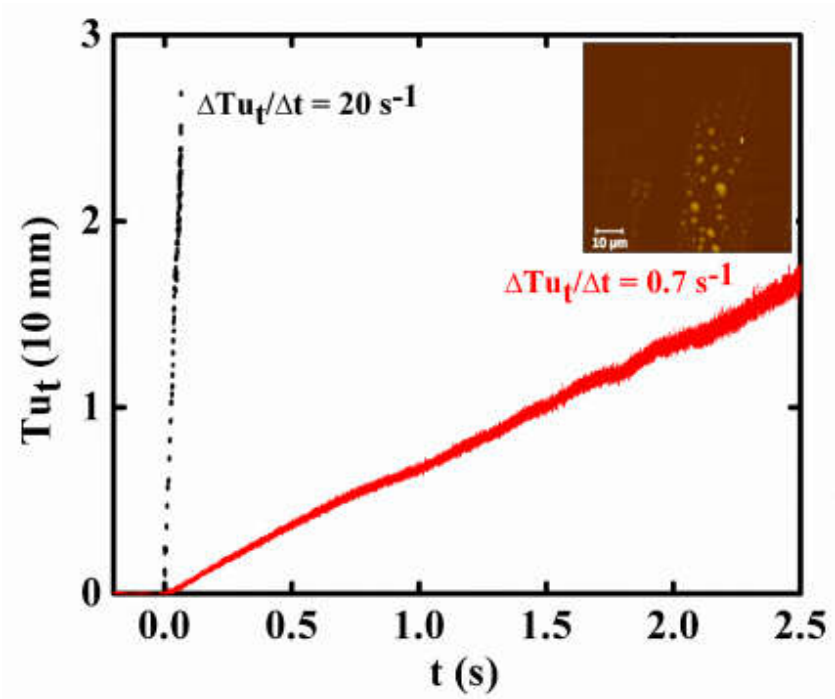

Figure 1. Time-dependent turbidity after the pulse for $c_{0}=5.3$ mol L-1, $l=10 \mathrm{~mm}, V=0.5 \mathrm{~mL}$ (in red). Inset: AFM image. The large white dots correspond to crystallites clusters. Dotted black line: signal observed in ref. 31 in the same solutions but with a tighter focusing of the laser pulse inducing a nuclei concentration 29 times higher (section 4.1). 
experiments than in ref. 31 and the energy deposited was less, so that the nuclei concentration and the turbidity rate were about 29 times less. However, for a given experiment, the kinetics signal and the crystal final size both result of the nuclei concentration produced and therefore they are univocally related.

Figure 1 presents a typical example of the kinetics signal and of the image of the corresponding final crystals, respectively. The concentration of the supersaturated solution is $c_{0}=5.3 \mathrm{~mol} \mathrm{~L}^{-1}(s=1.96)$, the volume in the cuvette is $V=0.5 \mathrm{~mL}$, and the optical path is $l=10 \mathrm{~mm}$. Very quickly after the pulse, the solution loses its transparency by the formation of the gel-like slurry of a high concentration of crystallites (Figure S2 b). On the AFM image, their shape appears as almost spherical.

The main feature of the kinetics observed under these conditions is that the turbidity is proportional to time up to the detection limit, as previously. ${ }^{31}$ However, under the same conditions of $s, l$, and sample volume $V$, the turbidity rate is significantly lower $\left(0.7 \mathrm{~s}^{-1}\right)$ and the final crystal diameter larger $(1850 \pm 100 \mathrm{~nm})$ than in that work $\left(20 \mathrm{~s}^{-1}\right.$ and $600 \mathrm{~nm}$, respectively). The growth process is basically an autocatalytic accretion reaction of molecules $M$ first on a supercritical nucleus growing into a crystallite $\mathrm{M}_{n}$ acting as the catalyst:

$\mathrm{M}_{n}+\mathrm{M} \leftrightarrow \mathrm{M}_{n+1}$

The growth rate is therefore controlled by the catalyst concentration increasing with the shock energy deposited by the pulse in the solution. Clearly, the energy deposited by pulses of the present setup is lower than previously. ${ }^{31}$ From section 4.1, the nuclei concentration is for example, under all equal other conditions, 30 times lower.

The final size of the crystals is reached when they are in equilibrium with the saturated solution. The final turbidity would correspond to $T u_{f}=\gamma_{T u} \times l \times\left(c_{0}-c_{s a t}\right)$. Note that, the upper detection limit being around $T u_{t}=2.5$, the measurement of $T u_{t}$ in cuvettes of $10 \mathrm{~mm}$ optical path is only a few $\%$ of $T u_{f}$.

It should be noted that, if the shock of the laser pulse is much lower than in current experiments, the concentration of crystals is much lower than above, they grow up to larger sizes, precipitate and continue to grow in the cuvette bottom, under non-homogeneous conditions. They acquire eventually the specific shape of long acicular millimetric $\mathrm{KNO}_{3}$ crystals (Figure S3a).

\subsection{Influence of the sample volume}

The laser beam is focused in a small volume where the supercritical nuclei are created and the convection caused by the shock spreads readily these germs throughout the sample volume which is large.

In order to estimate the influence of this early dispersion of the nuclei, we compared the turbidity rate with various volumes of the $5.5 \mathrm{~mol} \mathrm{~L}^{-1}$ supersaturated solution. Two types of cuvettes with different optical path were used and, because of their different geometries, the volume ranges studied were not the same (Figure 2).

Clearly, for both cuvettes, the turbidity rate decreases at increasing volume. Moreover, despite the uncertainty of measurements inherent to the crystallization phenomenon,
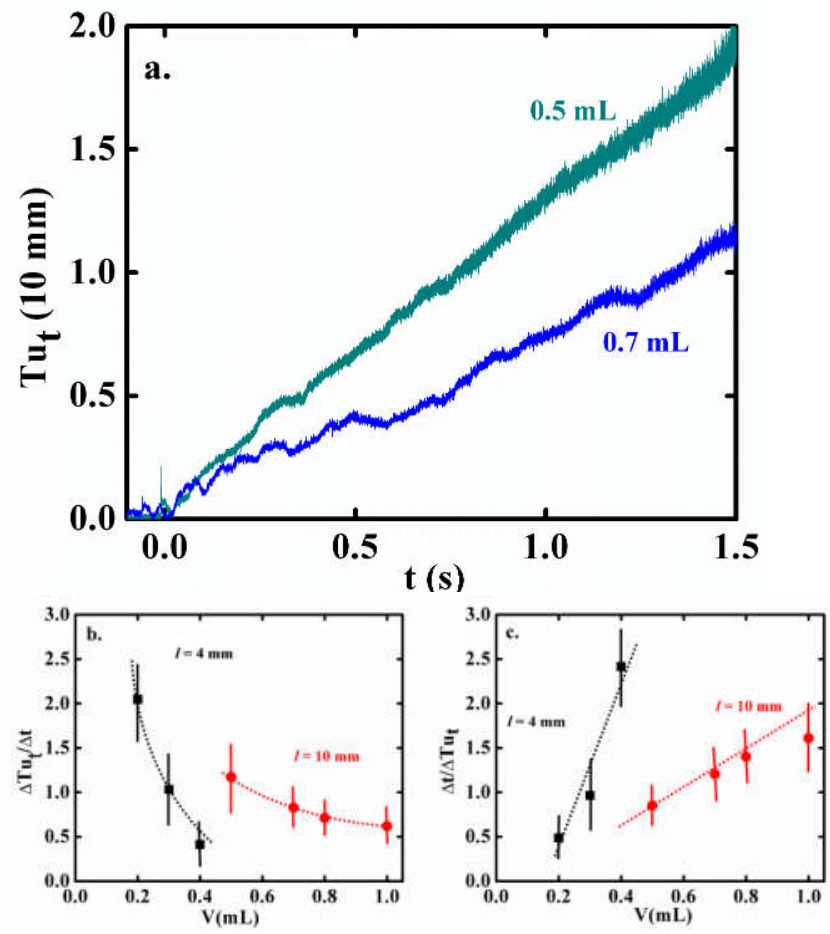

Figure 2. a. Turbidity increase for two sample volumes

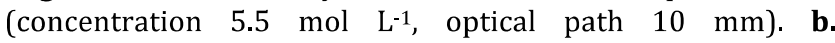
Dependence of the initial turbidity rate (average of 2 experiments) on the volume of the sample (in red: $l=10 \mathrm{~mm}$ in a $10 \times 10 \mathrm{~mm}^{2}$ cuvette; in black: $l=4 \mathrm{~mm}$ in a $10 \times 4 \mathrm{~mm}^{2}$ cuvette). c. Dependence of the reciprocal of the initial turbidity rate on the volume.

the rate roughly increases as a reciprocal of the volume (Figure 2c) as expected from an early dilution of nuclei by convection, immediately after the shock, from the same initial volume. This confirms that, when the turbidity is detected, the germs are already homogeneously distributed. The rates are also in the same ratio as the optical paths.

\subsection{Influence of the supersaturated concentration}

We observed the complete homogeneous formation of crystallites only for supersaturated concentrations equal to or higher than $c_{0}=4.3 \mathrm{~mol} \mathrm{~L}^{-1}$. Below this value, the crystallites grow up to large sizes, precipitate and continue to grow at the bottom of the cuvette (Figure SI2a). This concentration is higher than in the previous study, ${ }^{31}$ also confirming that the energy deposited by a single pulse is lower in the present setup than in the previous one. Figure 3 presents kinetics signals for the concentrations 4.9, 5.3, and $5.5 \mathrm{~mol} \mathrm{~L}^{-1}$ with the same sample volume and optical path. For each signal, the diameter of final crystals is measured by AFM (Figure 3 bottom). Higher is the concentration, faster is the acceleration. The size of the final crystals observed by AFM decreases slightly when the concentration increases. 


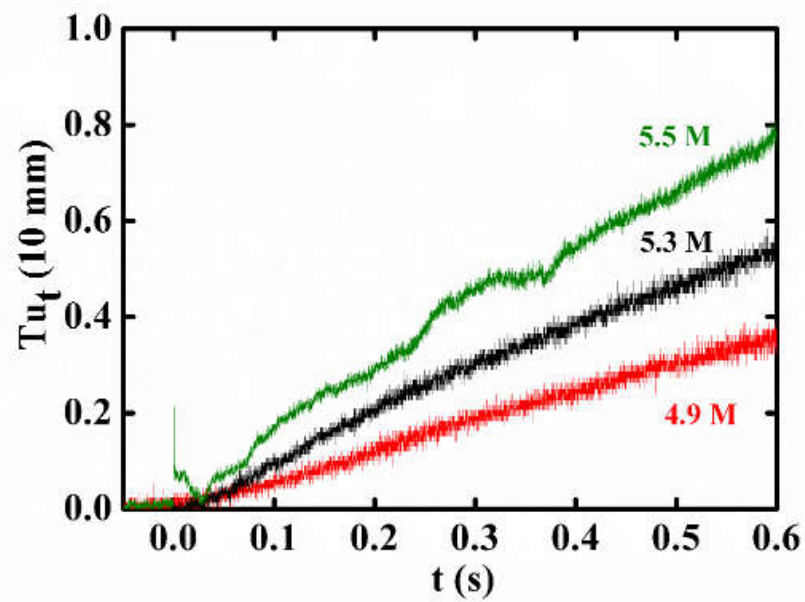

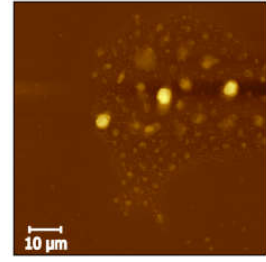
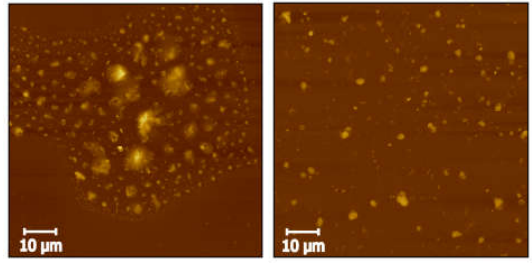

4.9 M

$5.5 \mathrm{M}$
Figure 3. Kinetic signals (top) and AFM images (bottom) at various concentrations with an optical path $l=10 \mathrm{~mm}$, and sample volume $V=0.5 \mathrm{~mL}$.

\subsection{Influence of the optical path}

At given concentration and volume, the growth phenomenon occurs independently of the optical path $l$. But the turbidity is measured proportionally to $l$. This influence can be studied only with cuvettes of different shape (Figure S1). In Figure 4, the supersaturation concentration was $c_{0}=5.5 \mathrm{~mol} \mathrm{~L}^{-1}$. The growth kinetics with a $4 \mathrm{~mm}$ or $2 \mathrm{~mm}$ optical path are observed with the same volume $V=0.3 \mathrm{~mL}$, and the signal for $10 \mathrm{~mm}$, obtained with a different volume $(V=0.5 \mathrm{~mL})$, was normalized according to the reciprocal law of Figure 2c. The turbidity rates are roughly proportional, as expected, to the optical paths $l$ (Figure $4 \mathrm{~b}$ ), despite the difference in the cuvette geometries which may influence the energy induced by the pulse and hence the nuclei concentration $c_{n}$. The optical path has no effect on the final shape and size of the crystals which depend only on $c_{0}$ and $c_{n}$.

When the same phenomenon of crystal growth occurs in cuvettes of still shorter optical path, i.e. $1 \mathrm{~mm}$ obtained by introducing a $1 \mathrm{~mm}$ spacer in the $10 \times 2 \mathrm{~mm}^{2}$ cuvette, the kinetic signal is observed during a longer time (Figure 5). In the early part, though much less intense than for longer optical paths, the turbidity $T u_{t, 1 \mathrm{~mm}}$ also increases proportionally to time as in the zoomed signal for $T u_{t, 10 \mathrm{~mm}}$. But, considering that the volume in these thin cuvettes is only $V=0.065 \mathrm{~mL}$, a normalization of the rate $\Delta T u_{t, 1 \mathrm{~mm}} / \Delta t$ as in Figure 2 for the same volume $0.3 \mathrm{~mL}$ would result in a value lower than the interpolated one (Figure 4). In fact, part of the energy of the shockwave is lost because it is also transferred to the walls of the cuvette and to the spacer. The final crystal sizes are also larger than in other cuvettes.
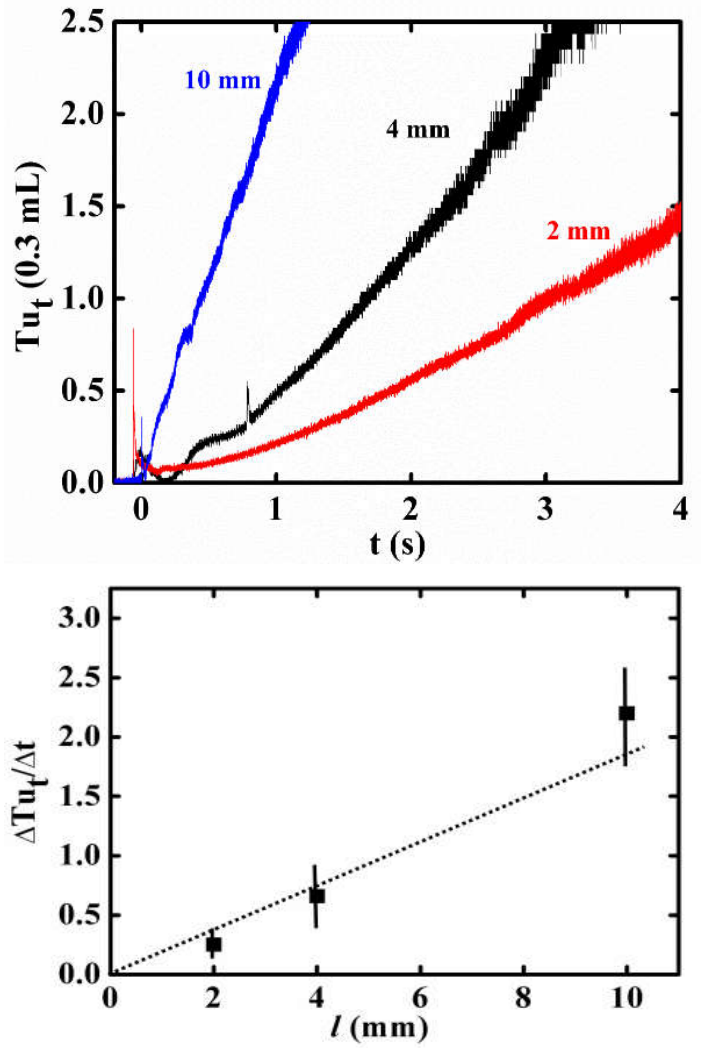

Figure 4. Influence of the optical path on the turbidity rate. Top: The initial supersaturation concentration is $c_{0}=5.5 \mathrm{~mol}$ $\mathrm{L}^{-1}$ and the sample volume is $V=0.3 \mathrm{~mL}$ The signal at $l=10$ $\mathrm{mm}(V=0.5 \mathrm{~mL})$ is normalized for the volume according to Figure 2. Bottom: Dependence of the turbidity rates (average of 2 experiments) on the optical path.

However, in the second part of the signal, the significant feature is that the turbidity rate is continuously accelerated. A similar phenomenon, but less marked, is observed at 4 and $2 \mathrm{~mm}$ (Figure 4). Because the nuclei concentration is fixed immediately after the pulse shock and the spontaneous nucleation is undetectable until minutes, it is excluded that the acceleration would result from an increase of the nuclei concentration. In contrast, during the growth, the number of possible accretion sites on each crystal may rapidly increase with the crystal area.

As for $10 \mathrm{~mm}$ optical path, higher is the concentration in the $1 \mathrm{~mm}$ cuvette, faster is the acceleration and also slightly smaller is the size of the final crystals observed by AFM (Figure 5 bottom for $l=1 \mathrm{~mm}: 2500 \mathrm{~nm}$ at $4.9 \mathrm{~mol} \mathrm{~L}^{-1}$ and $2300 \mathrm{~nm}$ at $5.5 \mathrm{~mol} \mathrm{~L}^{-1}$ ).

Figure 6 presents TEM images of the same sample at 5.3 mol $\mathrm{L}^{-1}$ as observed by AFM (Figure 5) but with a better resolution. Some isolated crystals and some other ones still glued together are often slightly elongated and sometimes display facets and two opposite pyramids with specific angles of $60^{\circ}$ as domes of orthorhombic nascent crystals (Figure S4). 


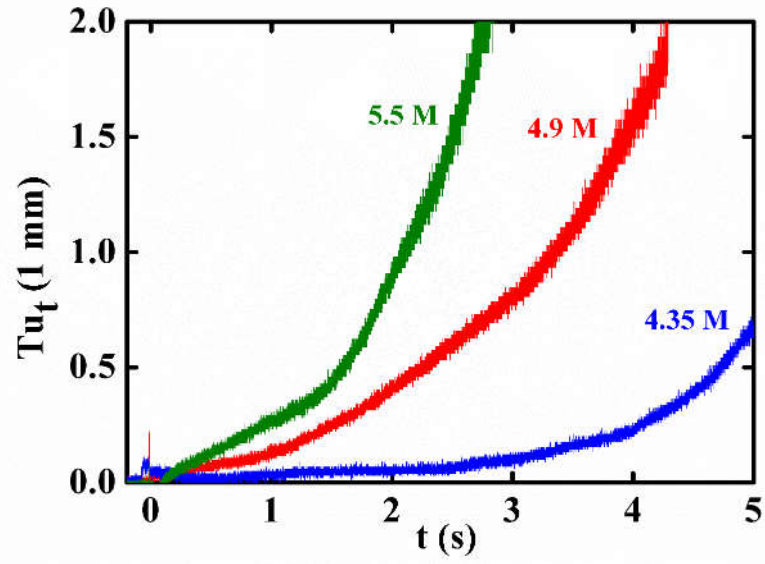

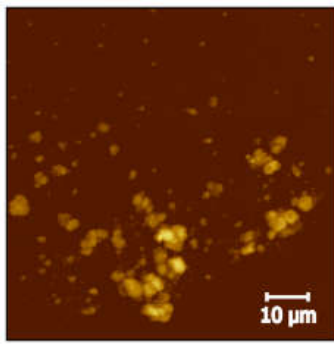

$5.5 \mathrm{M}$
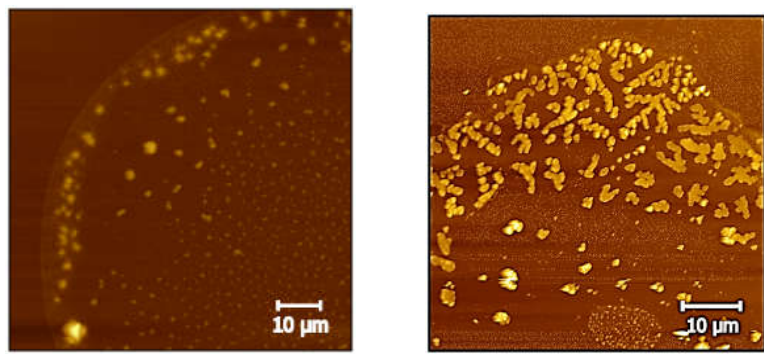

$4.9 \mathrm{M}$

Figure 5. Kinetic signals with an optical path of $l=1 \mathrm{~mm}$ at various concentrations (top). Corresponding AFM images of final crystals (bottom). On the right, areas of high density of crystallites where they are arranged in 2D supra-ordered dendrites and snowflake-like structures.

Note that the double pyramids are formed on a common (100) base only because the crystallites are freely moving in the solution, in contrast with the single pyramid of germ-mediated crystals. ${ }^{26}$ In average, the crystal diameter observed by AFM or TEM is close to $d=2000 \mathrm{~nm}$.

On certain slides prepared for AFM imaging, in areas where the slurry of crystals was dispersed before drying but with still a high concentration, we observe some supraordered two-dimensional structures of the crystallites. They are aligned in short lines and branches at angles close to $60^{\circ}$ or in snowflake-like structures (Figure 7 left). But these structures do not arise from twinning because they are constituted of individual monocrystals pre-formed in the suspension. Apparently, during the complete drying, where the contact between particles becomes possible, the quite homodisperse crystals are attracted by strongly attractive interactions along the (100) axis between their pyramidal summits and their (111) facets accordingly to
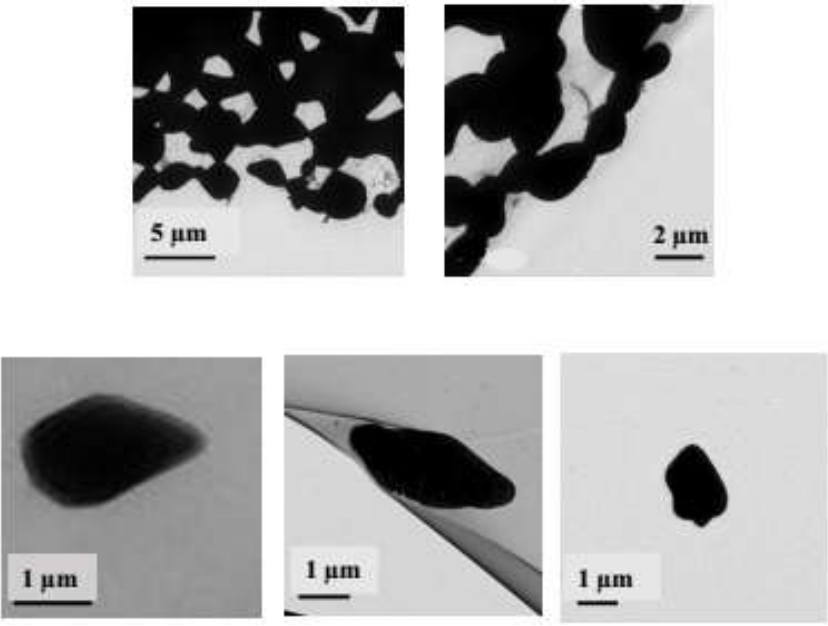

Figure 6. TEM images of final crystals at the supersaturation concentration with a sample volume $V=0.065 \mathrm{~mL}$ and an optical path $l=1 \mathrm{~mm}, c_{0}=4.9 \mathrm{~mol} \mathrm{~L}^{-1}$. Top: groups of crystals. Bottom: isolated crystals displaying pyramidal shapes, $c_{0}=$ $5.3 \mathrm{~mol} \mathrm{~L}^{-1}$.
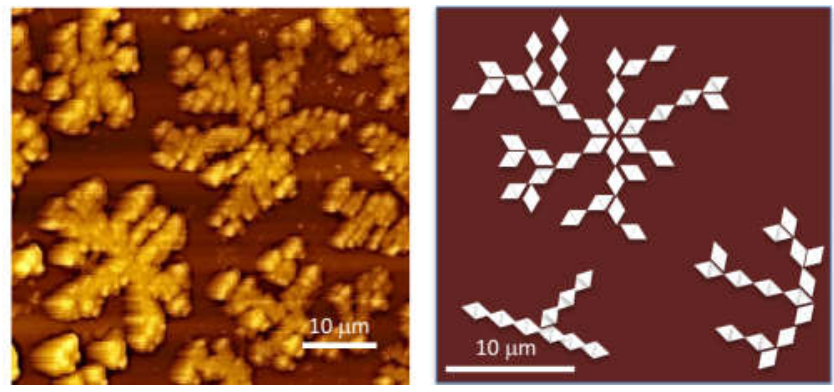

Figure 7. Left: Zoom of Figure 5 , bottom at $c_{0}=5.3 \mathrm{M}$ showing some hexagonal supra-structures, Right: Scheme of ordered supra-structures of $2 \mu \mathrm{m}$ bipyramidal crystals, controlled by the interaction between their summits along preferential orientations in straight lines of the 100 axis and between their (111) facets in dendrites at $60^{\circ}$.

favored orientations. In TEM images of crystal groups (Figure 6 top left), some of them seem also to be attracted by their summits along a common axis.

The scheme of Figure 7, right illustrates these orderings for bipyramidal crystals although the real ones are not all shaped so perfectly. Other supra-structures were observed for radiation-induced fcc crystals of $\mathrm{Ni}^{31}$ into necklaces or $\mathrm{Au}^{32}$ into (111) 2-D tiling, by HRTEM or STM microscopy, respectively.

\section{Homogeneous kinetics model of the growth}

The nuclei, then the crystals, are growing under homogeneous conditions. Hence, the analysis of the data may use the homogeneous kinetics model (HKM).

We observe that, under fixed conditions of supersaturation index, sample volume and optical path, the growth kinetics is markedly sensitive to the energy deposited (Figure 1). However, the measurement of both the observables of a given experiment, the kinetic signal 
and the final crystal size, are in univocal relation. This would enable us to determine the actual nuclei concentration and the rate constants of the process (see details below).

Clearly, the kinetics signals for $l=1 \mathrm{~mm}$ present two components (Figures 4 and 5): i) an initial component with a constant rate that is better observed with $10 \mathrm{~mm}$ of optical path, and ii) a second component with a continuously accelerated rate particularly observed with the very short optical path of $1 \mathrm{~mm}$. Though slower, such composite growth kinetics with induction time are often reported in the literature when crystals are produced by chemical reaction, antisolvent mixing, ... Because the Classical Nucleation Theory explains that the formation of supercritical nuclei is required before starting the growth into crystals, the initial increase is systematically assigned to the spontaneous formation of supercritical nuclei. However, in the present experiments, the supercritical nuclei are induced instantaneously during the laser pulse, without any chemical post-reaction and in large excess compared to the much slower and negligible spontaneous nucleation. Therefore, the assignment of the initial component to a nucleation-controlled process is excluded. Instead, we assign this initial process to a growth on the pulse-induced nuclei.

\subsection{Initial component}

a
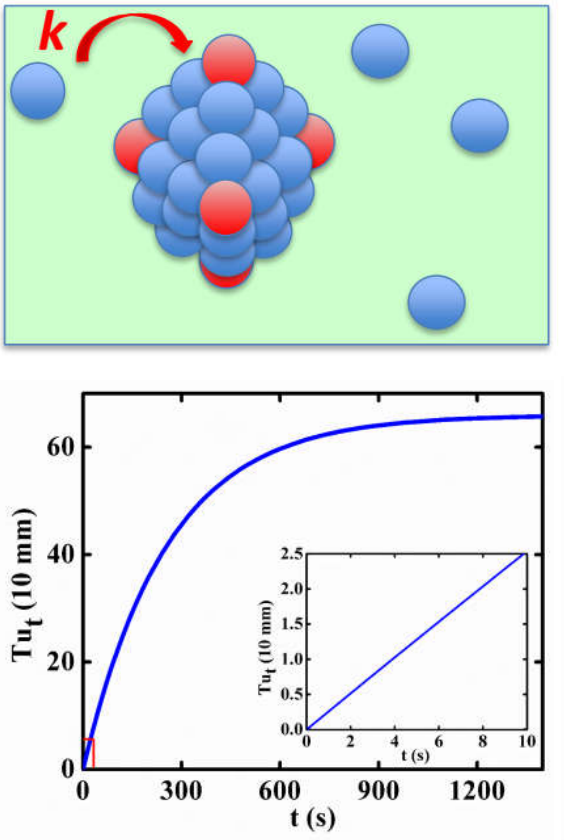

b
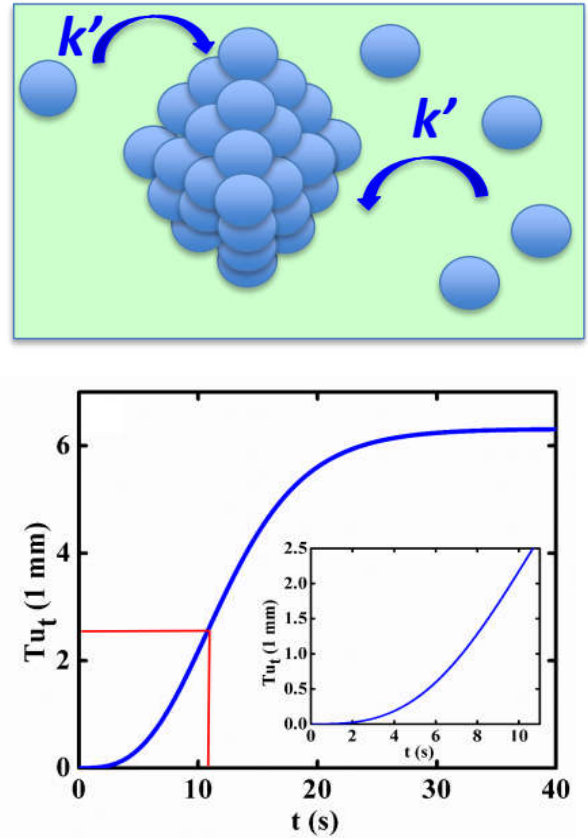

During the fast transition between the supersaturated solution and the crystals, the cascade of accretion processes of single dissolved molecule $\mathrm{M}$ on nuclei then crystals $\mathrm{M}_{n}$ growing into $\mathrm{M}_{n+1}$ with a rate constant $k$ coexists with a cascade of dissolution reactions of $\mathrm{M}_{n}$ into $\mathrm{M}_{n-1}$ with a rate constant $k \times c_{s a t}$ (equation 1). The concentration of growing crystals $\mathrm{M}_{n}$ is $c_{n, t}$ and each crystal contains $n_{t}$ molecules. According to the HKM model and as already stated, ${ }^{17}$ the derivative of the concentration at time $t$ of all the molecules incorporated in $\mathrm{M}_{n}$ crystals $\mathrm{d}\left(n_{t} \times\right.$ $\left.c_{n, t}\right) / \mathrm{d} t$ is overall equal to the derivative of the depletion of the molecules concentration in the solution $c_{t}$ and is proportional to $k \times\left(c_{t}-c_{s a t}\right)$ and to the concentration of the accretion sites ( $N$ per crystal) on the growing crystallites $N$ $\times c_{n, t}$ :

$\mathrm{d}\left(n_{t} \times c_{n, t}\right) / \mathrm{d} t=-\mathrm{d}\left(c_{t}\right) / \mathrm{d} t=k \times\left(c_{t}-c_{s a t}\right) \times N \times c_{n, t}$

and $\mathrm{d}\left(T u_{t}\right) / \mathrm{dt}=\gamma_{T u} \times I \times \mathrm{d}\left(n_{t} \times c_{n, t}\right) / \mathrm{d} t$

At very short times ( $\leq 1 \mathrm{~s})$, under any condition, $c_{t}$ is still close to $c_{0}$ and, as shown in particular for the optical path $l$ $=10 \mathrm{~mm}, 4 \mathrm{~mm}$, or even $1 \mathrm{~mm}$ (Figures 1-5), the turbidity is initially proportional to time, confirming also previous results for $10 \mathrm{~mm} .^{31}$ This zero-order kinetic law of the growth process implies several conclusions:

Figure 8. Autocatalytic accretion mechanism models (top) on a fixed concentration of nuclei $c_{n}$ and corresponding computerized turbidity increase up to the final equilibrium (bottom). $c_{0}=4.9 \mathrm{~mol} \mathrm{~L}^{-1}$. a. Accretion according to equation (4) only on the $N$ apex sites with a rate constant $N \times k=2.8 \times 10^{8} \mathrm{~L} \mathrm{~mol}^{-1} \mathrm{~s}^{-1}, l=10 \mathrm{~mm}$. b. Accretion on any site of apexes, edges and facets of a bipyramidal crystallite with

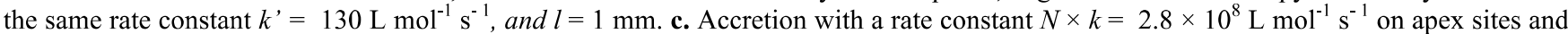
with a rate constant $k^{\prime}=130 \mathrm{~L} \mathrm{~mol}^{-1} \mathrm{~s}^{-1}$ on the other sites of the bipyramidal crystal, $l=1 \mathrm{~mm}$. Red frames correspond to the limit of the detection system $T u_{t}=2.3$ for $l=10$ or $1 \mathrm{~mm}$, and the insets represent this observed range.

c
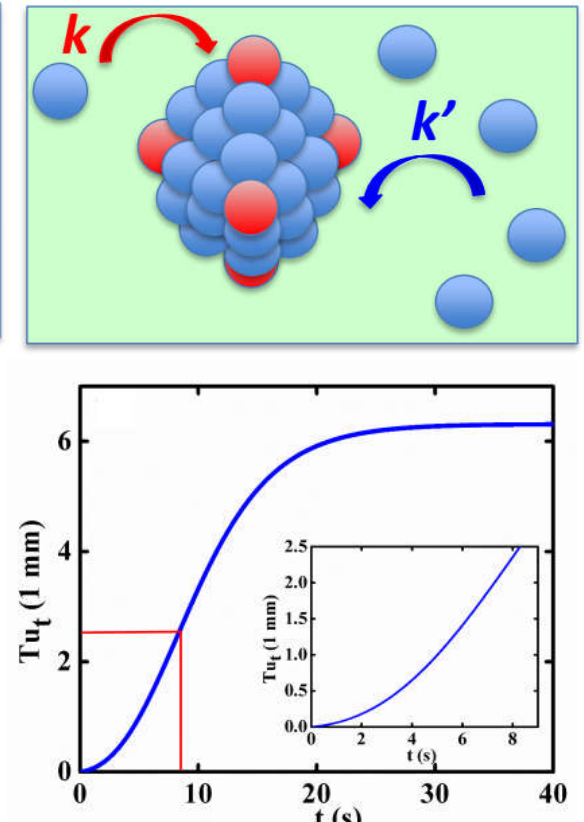
Table 1. Values of the rate constant $k$ calculated according to equation (6), and depending on the observables, the rate $\Delta T u / \Delta t$ and the final crystal diameter $d_{f}$. The optical path is $l=10 \mathrm{~mm}$, the sample volume is $V=0.5 \mathrm{~mL}$, and $N=6$.

\begin{tabular}{|c|c|c|c|c|c|c|c|c|}
\hline $\begin{array}{c}\boldsymbol{c}_{\boldsymbol{0}} \\
(\mathbf{M})\end{array}$ & $\begin{array}{c}\boldsymbol{c}_{\boldsymbol{\sigma}^{-}} \boldsymbol{c}_{\text {sat }} \\
(\mathbf{M})\end{array}$ & $\begin{array}{c}\boldsymbol{l} \\
(\mathbf{m m})\end{array}$ & $\begin{array}{c}\Delta \boldsymbol{T u} / \Delta \boldsymbol{t} \\
\left(\mathbf{s}^{-\mathbf{1}}\right)\end{array}$ & $\begin{array}{c}\boldsymbol{d}_{\boldsymbol{f}} \\
(\mathbf{n m})\end{array}$ & $\begin{array}{c}\boldsymbol{v}_{\text {crystal }} \\
\left(\mathbf{1 0}^{\mathbf{9}} \mathbf{n m}^{\mathbf{3}}\right)\end{array}$ & $\begin{array}{c}\boldsymbol{n}_{\boldsymbol{f}}\left(\mathbf{1 0}^{\mathbf{1 0}}\right. \\
\mathbf{m o l e c} / \mathbf{c r})\end{array}$ & $\begin{array}{c}\boldsymbol{c}_{\boldsymbol{n}} \\
\left(\mathbf{1 0}^{-11} \mathbf{M}\right)\end{array}$ & $\begin{array}{c}\boldsymbol{k} \\
\left(\mathbf{1 0}^{\mathbf{7}} \mathbf{M}^{-\mathbf{1}} \mathbf{s}^{-\mathbf{1}}\right)\end{array}$ \\
\hline 5.5 & 2.8 & 10 & 1.15 & 2000 & 4.2 & 5.3 & 5.3 & 4.5 \\
\hline 5.5 & 2.8 & 10 & 1.38 & 1920 & 3.7 & 4.7 & 6.0 & 4.8 \\
\hline 5.5 & 2.8 & 10 & 2.3 & 1800 & 3.1 & 3.8 & 7.3 & 6.6 \\
\hline 5.3 & 2.6 & 10 & 1.38 & 2000 & 4.2 & 5.3 & 4.9 & 5.9 \\
\hline 5.3 & 2.6 & 10 & 0.76 & 1800 & 3.1 & 3.8 & 6.8 & 2.3 \\
\hline 5.3 & 2.6 & 10 & 0.70 & 1850 & 3.3 & 4.2 & 6.2 & 2.3 \\
\hline 4.9 & 2.2 & 10 & 0.67 & 2000 & 4.2 & 5.3 & 4.2 & 3.3 \\
\hline
\end{tabular}

i) the concentration of growing crystallites $c_{n, t}$ is also constant as $c_{n}$, and equal to the concentration of initial supercritical nuclei created by the laser pulse. In fact, the increase of the crystal concentration by spontaneous creation of supercritical nuclei without laser pulse is negligible because it is much slower than the initial laser pulse-induced nucleation and the complete growth. Also, the crystal coalescence $\left(2 \mathrm{M}_{\mathrm{n}}\right.$ $\leftrightarrow \mathrm{M}_{2 \mathrm{n}}$ ) (or also called the particle attachment) ${ }^{33}$

is unlikely under our conditions, compared to the autocatalytic accretion of single molecules (reaction (1)), because $c_{n}$ is very low compared to $c_{0}$ and the concentration of nuclei and growing crystals $c_{n}$ does not decrease. Hence, the autocatalytic growth occurs at constant concentration $c_{n}$, only by accretion onto $\mathrm{M}_{n}$ of a supplementary molecule $\mathrm{M}$ from the supersaturated phase (reaction (1)) up to the end. ii) The zero-order law also implies that the turbidity coefficient $\gamma_{T u}$ is constant during the growth and independent of the crystal diameter. iii) Moreover, the proportional time-dependent turbidity implies that the number of accretion sites $N$ on each growing crystallite is also independent of its size in this time range. Among the different sites able to fix a next molecule, only the apexes $(N=6)$ present this feature and, at least within the short times range, they are thus the predominant accretion sites (Figure 8a bottom) and other ones can be neglected.

At longer times however, the solution concentration $c_{t}$ would progressively decrease from $c_{0}$ to the equilibrium concentration $c_{\text {sat. }}$ The general integration of (1) with a constant value of $N \times c_{n} \times k$ would lead to an exponential decrease of $c_{t}$ to $c_{s a t}$ and to an exponential increase of $\left(n_{t} \times\right.$ $\left.c_{n}\right)$ up to $\left(c_{0}-c_{s a t}\right)$. Thus:

$T u_{t}=\gamma_{T u} \times l \times\left(n_{t} \times c_{n}\right)=\gamma_{T u} \times l \times c_{n} \times\left(c_{0}-c_{s a t}\right) \times(1-\exp (-N \times$ $\left.c_{n} \times k \times t\right)$ )

In Figure 8a, the turbidity rate would decelerate up to the plateau bottom which corresponds to the complete transition of the supersaturated solution into crystals with the final size and the saturated solution:

$T u_{f}=\gamma_{T u} \times l \times\left(c_{0}-c_{s a t}\right)$
Note that with $l=10 \mathrm{~mm}$ and $c_{0}=4.9 \mathrm{~mol} \mathrm{~L}^{1}$, the upper limit of the detected turbidity) is $T u_{t}=2.5$ (in red in the insets of Figure 8 , bottom), that is only $3.5 \%$ of the final value $T u_{f}=66$ for $l=10 \mathrm{~mm}$ and $35 \%$ of $T u_{f}=66$ for $l=1$ $\mathrm{mm}$.

The initial part of the signal $\left(c_{t} \approx c_{0}\right)$ is indeed proportional to time as in the Inset of Figure $8 \mathrm{a}$, bottom, with the constant rate:

$\mathrm{d}\left(T u_{t}\right) / \mathrm{d} t=\gamma_{T u} \times l \times\left(c_{0}-C_{s a t}\right) \times N \times k \times c_{n}$

This implies the very important conclusion that the coefficient of turbidity per molecule is constant and is independent in particular of the particle diameter. This is demonstrated by the proportional increase of the turbidity which is found systematically with the optical path $l=10$ $\mathrm{mm}$. Moreover, we may compare the nuclei formation presented in Figure 1 under same conditions except the laser focusing producing very different nuclei concentrations $c_{n}$.

The final number of molecules per crystal $n_{f}$ is derived from the final crystal diameter $d_{f}$, the specific mass $\rho$ of $\mathrm{KNO}_{3}$, and the Avogadro number $N_{A}$ :

$n_{f}=\pi \times d_{f}^{3} \times N_{A} \times \rho / 6 M_{w}$

The constant concentration of growing crystals $c_{n}$ is:

$c_{n}=\left(c_{0}-c_{s a t}\right) / n_{f}=6 M_{w}\left(c_{0}-c_{s a t}\right) / \pi \times N_{A} \times \rho \times d_{f}^{3}$

According to (6) and (8), the turbidity rate increases as $1 / d_{f}^{3}$. In Figure 1 , the ratio of the turbidity rates of the signals is $20 / 0.7=28.6$, whereas the ratio of $1 / d_{f}^{3}$ is 29.2 , confirming that the coefficient per molecule $\gamma_{T u}$ has the same value in both cases even if the ranges of the particle 
diameter are different (up to $200 \mathrm{~nm} v s$ up to $600 \mathrm{~nm}$ in the first component). (See also SI).

According to the experimental results and to relation (6), the rate at given optical path $l$ and supersaturated concentration $c_{0}$ is indeed proportional to the nuclei concentration $c_{n}$, that varies as the reciprocal of the sample volume (Figure $2 \mathrm{c}$ ), and, at given sample volume and $c_{0}$, the rate is proportional to the optical path $l$ (Figure 4 ). The rate increases with the supersaturated concentration $c_{0}$ (Figure 3). However, the variation is complex as already observed in the pioneering study ${ }^{24}$ because the laser energy deposition, and hence also $c_{n}$, depend on $c_{0}$.

Table 1 presents for a series of experiments the corresponding values of the initial slope of the kinetic signal and of the final diameter of the crystal, both depending univocally on the shock energy deposited.

In Table 1 , the number of molecules per crystal $n_{f}$ is calculated from $d_{f}$ by relation (7) and $c_{n}$ by relation (8). Note that the uncertainty on the final diameter is $\pm 10 \%$, and that on the values of $n_{f}$ and $c_{n}$ (arising from $d_{f}^{3}$ ) is \pm $30 \%$. The uncertainty on the turbidity rate $\Delta T u / \Delta \mathrm{t}$ is \pm $10 \%$. Hence the uncertainty on the $k$ value is $\pm 40 \%$.

The rate constant of accretion $k$ is found independent of the experimental conditions. The mean value is $k=(4.7 \pm$ 2) $\times 10^{7} \mathrm{~L} \mathrm{~mol}^{-1} \mathrm{~s}^{-1}$. It is in fair agreement with the value previously published (with $N=6$ ), ${ }^{31}$ although the conditions of the energy transfer to the sample are now much different as shown by the concentration of nuclei $c_{n}$. The $k$ value is much lower than a diffusion-controlled reaction rate constant in water, indicating that some activation energy is due to the reorganization of the molecule during the accretion. Note that the molecules transferred to the summits by direct accretion may not stay there but may diffuse to the next sites and contribute indirectly to the mean growth of the edges and facets. But direct accretion on the sites of the edges and facets is kinetically negligible during this first component.

\subsection{Second component}

For an optical path as short as $l=1 \mathrm{~mm}$ (Figures 5 top and $8 \mathrm{~b}$ and c, bottom), $T u_{t 1 \mathrm{~mm}}$ is much lower than $T u_{t} 10 \mathrm{~mm}$. Therefore, according to relation (6), the initial rate is low and the turbidity increase is observed during a longer time before reaching the detection limit. It is worthy to note that the shape of the experimental kinetics obtained is significantly different from the exponential of Figure $8 \mathrm{a}$, bottom. In contrast, after the slow initial increase in Figure 5 , the rate of the experimental increase is continuously accelerated, implying that at longer times other accretion sites are now efficiently active.
If we assume that the accretion occurs isotropically with the same rate constant $k^{\prime}$ not only on the apexes but also on the sites of the edges and the facets (Figure $8 \mathrm{~b}$ ), the number of superficial sites $N_{t}^{\prime}$ per crystal increases rapidly with the size and $n_{t}$ during the growth. If the crystal shape is considered as perfectly bipyramidal with equilateral facets (111) as observed by TEM (Figure 6, bottom), the superficial site number increases as $N_{t}^{\prime}=2.8 n_{t}{ }^{2 / 3}$ (Figure $8 \mathrm{~b})$. The rate constant $k^{\prime}$ is assigned mostly to the (111) facet sites because, except for crystals precipitated at very low laser energy and for much longer times, no long acicular shape was observed. Under the current laser pulse conditions, the concentration of nuclei $c_{n}$ is high and the supersaturated molecule concentration is exhausted before the crystal size exceeds $d_{f} \approx 2 \mu \mathrm{m}$.

The rate of the turbidity increase is thus:

$\mathrm{d}\left(T u_{t}\right) / \mathrm{d} t=\gamma_{T u} \times l \times \mathrm{d}\left(n_{t} \times c_{n}\right) / \mathrm{d} t=\gamma_{T u} \times l \times c_{n} \times k^{\prime} \times 2.8 \times$ $n_{t}^{2 / 3} \times\left(c_{0}-c_{s a t}-\left(n_{t} \times c_{n}\right)\right)$

The time-evolution of $T u_{t}$ resulting from the numerical integration of (9) is presented in Figure $8 \mathrm{~b}$ bottom. It is worthy to note that at $t=0$ where $n_{t}=0$, the derivative (9) is also equal to zero. The tangent at the origin $t=0$ of the simulated signal is thus the abscissae axis (Figure $8 \mathrm{~b}$ bottom, Inset). This particular feature, based on an isotropic accretion of molecules on any site of the crystallite, is in marked disagreement with the experimental observations of an initial linear increase of the turbidity at short times (Figures 1-5). Note that, with an assumption of nascent crystallites being of any other shape than bipyramidal, the derivative would be also in $n_{t^{2 / 3}}$ and equal as well to zero at $t=0$.

Consequently, we should consider that the accretion is definitely an anisotropic process, with at least two different rates, that is $k$ on a fixed number of sites (the apexes) which is responsible of the linear increase, and $k^{\prime}$ on the other sites (of edges and facets), the number of which increases rapidly with the growing diameter of the particles and which are responsible of the accelerated increase (Figure 8c). In this hypothesis of two distinct accretion rate constants on apexes and on the other sites of a bipyramidal crystal, the turbidity derivative is:

$\mathrm{d}\left(T u_{t}\right) / \mathrm{d} t=\gamma_{T u} \times l \times \mathrm{d}\left(n_{t} \times c_{n}\right) / \mathrm{d} t=\gamma_{T u} \times c_{n} \times\left(N \times k+2.8 \times k^{\prime}\right.$ $\left.\times n_{t}^{2 / 3}\right) \times\left(c_{0}-c_{s a t}-\left(n_{t} \times c_{n}\right)\right)$

The numerical integration of equation (10) is presented in Figure 8c, bottom. The value of $T u_{f}$ at the plateau is independent of the rates: $T u_{f}=\gamma_{T u} \times l \times\left(c_{0}-c_{s a t}\right)$. Because the optical path is only $l=1 \mathrm{~mm}$, the upper detection limit of $T u_{f}$ 
Table 2. Adjusted values of the rate constant $k^{\prime}$ for various experimental kinetic signals and corresponding final diameters $d_{f}$ (optical path $l=1 \mathrm{~mm}, k=(4.7 \pm 2) \times 10^{7} \mathrm{~L} \mathrm{~mol}^{-1} \mathrm{~s}^{-1}, N=6, N_{t}^{\prime}=4 n_{t}{ }^{2 / 3}$ ).

\begin{tabular}{|c|c|c|c|c|c|c|c|}
\hline $\begin{array}{c}c_{0} \\
(\mathrm{M})\end{array}$ & $\begin{array}{c}c_{0}-c_{s a t} \\
\text { (M) }\end{array}$ & $\begin{array}{c}I \\
(\mathrm{~mm})\end{array}$ & $\begin{array}{c}d_{f} \\
(\mathrm{~nm})\end{array}$ & $\begin{array}{c}V_{\text {crystal }} \\
\left(10^{9}\right. \\
\left.\mathrm{nm}^{3}\right)\end{array}$ & $\begin{array}{c}n_{f}\left(10^{10}\right. \\
\text { molec./crystal }\end{array}$ & $\begin{array}{c}c_{n} \\
\left(10^{-11} \mathrm{M}\right)\end{array}$ & $\begin{array}{c}k^{\prime} \\
\left(M^{-1} s^{-1}\right)\end{array}$ \\
\hline 5.5 & 2.8 & 1 & 2210 & 5.6 & 7.1 & 3.9 & 34 \\
\hline 5.5 & 2.8 & 1 & 2700 & 10 & 13 & 2.2 & 111 \\
\hline 5.3 & 2.6 & 1 & 1950 & 3.9 & 4.9 & 5.3 & 127 \\
\hline 4.9 & 2.2 & 1 & 2100 & 4.8 & 6.1 & 3.6 & 48 \\
\hline 4.9 & 2.2 & 1 & 2900 & 13 & 16 & 1.4 & 192 \\
\hline
\end{tabular}

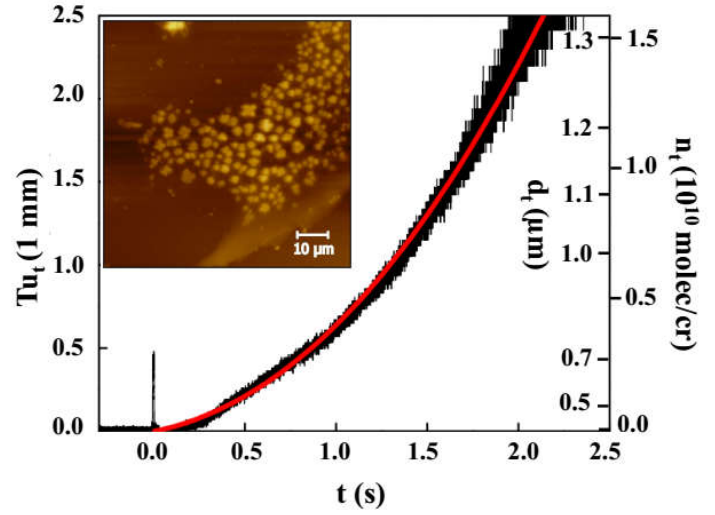

Figure 9. Time-evolution of the turbidity in $5.3 \mathrm{~mol} \mathrm{~L}$ ${ }^{1}$ solution $(l=1 \mathrm{~mm})$ (in blue). The final diameter of the crystals is $d_{f}=1.95 \mu \mathrm{m}$. The simulated curve (in red) is obtained with $\mathrm{Tu}_{f, 1 \mathrm{~mm}}=7.8, N \times k=2.8 \times 10^{8} \mathrm{~L}$ $\mathrm{mol}^{-1} \mathrm{~s}^{-1}, c_{n}=5.3 \times 10^{-11} \mathrm{~mol} \mathrm{~L}^{-1}$, and the adjusted value of $k^{\prime}=127 \mathrm{~L} \mathrm{~mol}^{-1} \mathrm{~s}^{-1}$. The values of $n_{t}$ and $d_{t}$ are given on the right ordinate axis.

corresponds now to almost $35 \%$ of $T u_{f}$. The specific feature of the model kinetic signal in Figure 8c, bottom is together to increase linearly at short time in the first component (and not to be tangent to the abscissae axis), and to be continuously accelerated in the second part.

The adjustment of the simulated signals with the experimental signals consists in taking the $N \times k$ value determined above as $N \times k=(2.8 \pm 1.2) \times 10^{8}$ $\mathrm{L} \mathrm{mol}^{-1} \mathrm{~s}^{-1}$, then in taking for each signal the value of the crystal concentration $c_{n}$ as calculated from the corresponding measured diameter $d_{f}$ (equation 7) and in adjusting the value of $k^{\prime}$. An example of adjustment between the experimental and simulated signals is presented in Figure 9. In this example, the final diameter of crystals is $d_{f}=1.95$ $\mu \mathrm{m}$. In Figure 9 , the value of $n_{t}$, that is given on the right axis of ordinates, is proportional to $T u_{t}$, but $d_{t}$ increases as $\sqrt[3]{T u_{t}}$. For example, at the end of the first linear component, the diameter is $d_{2 s}=720 \mathrm{~nm}$

Table 2 presents the results of the adjusted values of $k^{\prime}$ for various samples with different concentrations and signals of Figure 10. The
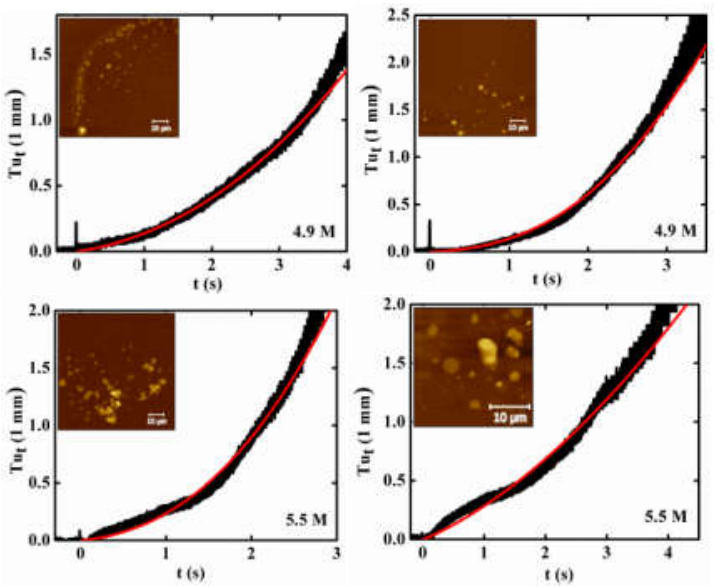

Figure 10: Adjustment of the time-evolution of $\mathrm{Tu}_{t}$ according to the integration of equation (9) for some selected experimental kinetic signals $(l=1 \mathrm{~mm})$. The accretion of supersaturated molecules is assumed to occur anisotropically with the accretion rate constant $N \times k=2.8 \times 10^{8} \mathrm{~L} \mathrm{~mol}^{-1} \mathrm{~s}^{-1}$ on the sites of apexes $(N=$ $6)$ and with the rate constant $k^{\prime}$ on the other sites. The value of the crystal concentration $c_{n}$ is calculated from the observed final diameter $d_{f}$. The adjusted values of the accretion rate constant $k^{\prime}$ on the sites of edges and facets are presented in Table 2 .

uncertainty on $k^{\prime}$ results from that on $k(40 \%), c_{n}$ $(30 \%)$ and on the kinetics adjustment. In spite of this uncertainty, the adjusted value of the rate constant of molecules accretion on facets and edges is in fair agreement for all samples and the mean value is: $k^{\prime}=(100 \pm 70) \mathrm{L} \mathrm{mol}^{-1} \mathrm{~s}^{-1}$. The value is several orders of magnitude lower than that of $k$, but the number of sites on the facets and edges increases rapidly with the diameter, thus explaining that the accretion rate of the crystal becomes predominant.

Nevertheless, the probability of accretion on a single site of an edge or facet is highly lower than on an apex $\left(k^{\prime} / k \approx 10^{-5}\right)$. Hence the growth is highly anisotropic and is favored on the apex. If the supersaturated concentration of the surrounding 
solution did not progressively decrease down to $c_{\text {sat }}$, the growing crystals would acquire an elongated shape. Actually, in some experiments where the concentration of nuclei is very low because the supersaturation index is too low, the growth may continue up to large sizes and we may even observe the formation of long acicular crystals.

It is interesting to compare the structure of crystals of $\mathrm{KNO}_{3}$ grown by the single germmediated method at low and stationary supersaturated concentration ${ }^{26}$ with the structure of present multiple crystals growing under homogeneous conditions on laser pulse-induced nuclei in highly supersaturated solutions. In both studies, the growth starts by the building of pyramids with (111) faces on a (100) base, specific of the rhombohedral structure. The kinetics of the germ-mediated growth was observed for hours and the front shifts on various faces was evaluated beyond $\approx 200 \mu \mathrm{m} .{ }^{25}$ However, the present study of homogeneous kinetics is focused on an early period of the growth that stops at around $5 \mathrm{~s}\left(d_{f} \leq 2\right.$ $\mu \mathrm{m})$. It let appear an early constant rate assigned to the accretion on apexes, before an accelerated rate increasing with the superficial site concentration, assigned to the accretion on the (111) faces.

\section{CONCLUSION}

Under conditions of highly supersaturated solutions, a single laser pulse shockwave induces instantaneously a great number of supercritical nuclei. Thus, the steps of nucleation and of the following growth occur in separate time ranges. Because each nucleus collects a small part of the surrounding molecules and because, after rapidly exhausting the supersaturated molecules, they never grow enough to precipitate, the increase of their turbidity obeys homogeneous kinetics. Hence, this experimental approach gives access to a quantitative study of the crystal growth, in particular to the very early part by using $10 \mathrm{~mm}$ optical path and to the longer part of the total growth by using $1 \mathrm{~mm}$ optical path. A detailed analysis of these kinetics signals and measurements of the corresponding final crystal size at various optical paths, sample volumes or supersaturation indexes enables us to conclude to an anisotropic growth of $\mathrm{KNO}_{3}$ crystallites with a much higher accretion rate constant of molecules on the apex sites of the crystals than on the other sites (edges and facets). The concentration of nuclei and the rate constants are evaluated.

\author{
ASSOCIATED CONTENT \\ Supporting Information \\ Materials and Methods, Figures S1-S4. This \\ ACS Paragon Plus Environment
}

Internet at http:// pubs.acs.org/.

\author{
AUTHOR INFORMATION \\ Corresponding Author \\ *E-mail: jacqueline.belloni@universite-paris- \\ saclay.fr.
}

\section{Notes}

The authors declare no competing financial interest.

\section{ACKNOWLEDGMENTS}

The authors thank warmly Audrey Gayral, Jean Philippe Larbre and Pierre Jeunesse for her helpful collaboration in constructing and maintaining the laser and optical spectroscopy devices. S.A-G. is grateful to the Lebanese University for the scholarship during 2017-2020. 
(1) Chernov, A. A., Ed. Modern Crystallography. Crystal Growth; Springer: Berlin, 1984; Vol. III.

(1) Tieng, S.; Azouani, R.; Chhor, K.; Kanaev, A. Nucleation-Growth of $\mathrm{TiO}_{2}$ Nanoparticles Doped with Iron Acetylacetonate, J. Phys. Chem. C 2011, 115, 52445250 .

(2) Garetz, B. A.; Aber, J. E.; Goddard, N. L.; Young, R. G.; Myerson, A. S. Nonphotochemical, PolarizationDependent, Laser-Induced Nucleation in Supersaturated Aqueous Urea Solutions, Phys. Rev. Lett. 1996, 77, 34753476.

(3) Zaccaro, J.; Matic, J.; Myerson, A.S.; Garetz B.A. Nonphotochemical, Laser-Induced Nucleation of Supersaturated Aqueous Glycine Produces Unexpected Gamma-Polymorph, Cryst. Growth Des., 2001, 1, 5.

(5) Sun, X.; Garetz, B. A.; Myerson, A. S. Supersaturation and Polarization Dependence of Polymorph Control in the Non Photochemical Laser-Induced Nucleation (NPLIN) of Aqueous Glycine Solutions, Cryst. Growth Des. 2006, 6, 684-689.

(5) Alexander, A. J.; Camp, P. J., Single Pulse, Single Crystal Laser-Induced Nucleation of Potassium Chloride, Cryst. Growth Des. 2009, 9, 958-963.

(6) Sugiyama, T.; Adachi, T.; Masuhara, H. Crystal Growth of Glycine Controlled by a Focused CW Near-infrared Laser Beam, Chem. Lett. 2009, 38, 482-483.

( 7 ) Clair B., Ikni A., Scouflaire P., Quemener V., Spasojevic-de Biré A., A New Experimental Setup for High-Throughput Controlled Non-Photochemical LaserInduced Nucleation: Application to Glycine Crystallization, J. App. Cryst., 2014, 47, 1252-1260.

(8) Ikni A., Clair B., Scouflaire P., Veesler S., Gillet J-M., El Hassan N., Dumas F., Spasojevic-de Biré A., Experimental Demonstration of the Carbamazepine Crystallization From Non-Photochemical LASER-Induced Nucleation in Acetonitrile and Methanol, Cryst. Growth Des. 2014, 42, 3286-3299.

(9) Alexander, A. J.; Camp, P. J., Non-Photochemical LaserInduced Nucleation. J. Chem. Phys. 2019, 150, 040901.

(10) Yoshikawa, H.Y.; Hosokawa,Y.; Masuhara, H. Spatial control of urea crystal Growth by focused femtosecond laser irradiation. Cryst. Growth Des. 2006, 6, 302-305.

( 11 ) Nakamura, K.; Hosokawa, Y.; Masuhara, H. Anthracene Crystallization Induced by Single-Shot Femtosecond Laser Irradiation: Experimental Evidence for the Important Role of Bubbles, Cryst. Growth Des. 2007, 7, 885-889.

(12) Fischer, A. ; Pagni, R.M. ; Compton, R.N. ; Kondepudi, D. Laser Induced Crystallization, in Jena P.; Castleman, A.W. (Eds) Nanoclusters- A bridge across disciplines. Elsevier, 2010, vol. 1, ch. 8, 343-364.

(13) Soare, A.; Dijkink, R.; Pascual, M. R.; Sun, C.; Cains, P. W.; Lohse, D.; Stankiewicz, A. I.; Kramer, H. J. M. Crystal Nucleation by Laser-Induced Cavitation; Cryst. Growth Des. 2011, 11, 2311-2316.

(14) Wang, Shubo; Wang, Sumei; Jiang, Lan; Wang, Mengmeng; Wei, Yuyin; Sun, Jiaxin; Zhan, Shenghua; Li, Xin; Qu, Liangti. Polymorph-Controlled Crystallization of Acetaminophen through Femtosecond Laser Irradiation. Cryst. Growth Des. 2019, 19, 3265-3271.

( 15 ) Nohan, N.M.; Fischer, A.; Graves, B.; Bolorizadeh,M.; Kendepudi, D.; Compton, R.N. Laser Shock-Wave Induced Crystallization. Cryst. Growth Des. 2017, 17, 576-581.

(16) Lee, B. S.; Burr, G. W.; Shelby, R. Mri Rapux S.; Rettner, C. T.; Bogle, S. N.; Damarwikarta, K.; Bishop, S. G.;
Abelson, J. R. Observation of the Role of Subcritical Nuclei in Crystallization of a Glassy Solid, Science 2009, 326, 980-984.

(17) McAnany, S.D.; Veenhuizen, K.; Nolan, D.A.; Aitken, B.G.; Dierolf, V.; Jain, H. Challenges of Laser-Induced Single-Crystal Growth in Glass: Incongruent Matrix Composition and Laser Scanning Rate. Cryst. Growth Des. 2019, 19, 4489-4497.

(18) Cao, J.; Mazerolles, L.; Lancry, M.; Brisset, F.; Poumellec, B. Modifications in Lithium Niobium Silicate Glass by Femtosecond Laser Direct Writing: Morphology, Crystallization, and Nanostructure. J. Opt. Soc. Am. $B$, 2017, 34, 160-168.

(19) Zeldovich, Ya. B. On the Theory of New Phase Formation : Cavitation. Acta Physicochim. (Russian) 1943, $18,1-22$.

(20) L'Hermite, J.-M. Growth and Melting of Droplets in Cold Vapors, Phys. Rev. E 2009, 80, 051602.

(21) Belloni, J.; Pernot, P., Role of Nuclearity-Dependent Properties of Small Clusters in the Nucleation Process and Thermal Hysteresis at the Phase Transition, J. Phys. Chem. B 2003, 107, 7299-7302.

(22) Quigley, D.; Rodger, P.M. Free Energy and Structure of Calcium Carbonate Nanoparticles During Early Stages of Crystallization, J. Chem. Phys. 2008, 128, 221101.

(23) Niu, H.; Piaggi, P. M.; Invernizzi, M.; Parrinello, M., Molecular Dynamics Simulations of Liquid Silica Crystallization, Proc. Nat. Ac. Sci. 2018, 115, 5348-5352.

(24) Leroudier, J.; Zaccaro, J.; Ildefonso, M.; Veesler, S.; Baruchel, J.; Ibanez, A. Nucleation Control and Rapid Growth of KDP Crystals in Stationary Conditions. Cryst. Growth Des. 2011, 11, 2592-2598.

(25) Manomenova, V.; Rodneva, E.; Baskakova, S.; Voloshin, A. Growth of KDP:Fe3+ Crystals from Oversaturated Aqueous Solutions. Crystals 2019, 9, 646.

(26) Rolfs, J.; Lacmann, R.; Kipp, S. Crystallization of Potassium Nitrate $\left(\mathrm{KNO}_{3}\right)$ in Aqueous Solution I. Growth Kinetics of the Pure System, J. Cryst. Growth, 1997, 171, 174-182.

(27) Kipp, S.; Kämmer, S.; Lacmann, R.; Rolfs, J.; Tanneberger, U.; Beckmann, W. Imaging Crystal Growth Features Using Scanning Force Microscopy (SFM). Cryst. Res. Technol. 1994, 29, 1005-1011.

(28) Hong, M.; Moreland, K.T.; Chen, J.J; Teng, H.H.; Thalmann, R.; Effect of Proteins Fetuin A, Osteopontin, and Otoconin 90 on the Nucleation and Growth of Calcite. Cryst. Growth Des. 2015, 15, 129-136.

(29) Fieury, B.; Neouze, M.A., Guigner, J.M.; Menguy, N.; Spalla, O., Gacoin, T., Carrière, D. Amorphous to Crystal Conversion as a Mechanism Governing the Structure of Luminescent YVO4:Eu Nanoparticles. ACS Nano 2014, 8 , 2602-2608.

(30) Jacob, J. A. ; Sorgues, S. ; Dazzi, A. ; Mostafavi, M., Belloni, J. Homogeneous Nucleation-Growth Induced by Single Laser Pulse in Supersaturated Solutions. Cryst. Growth Des. 2012, 12, 5980-5985.

(31) Belloni, J.; Mostafavi, M.; Remita, H.; Marignier, J.-L.; Delcourt, M.-O. Radiation-Induced Synthesis of Monoand Multi-Metallic Clusters and Nanocolloids. New J. Chem., 1998, 1239-1255.

( 32 ) Belloni, J.; Remita, H. Metal Clusters and Nanomaterials. In Radiation Chemistry: From basics to applications in material and life sciences. SpotheimMaurizot, M.; Douki, T.; Mostafavi, M.; Belloni, J. (eds), EDP Sciences, 2008, 101-120.

(33) De Yoreo, J.J.; Gilbert P.U.P.A.; Sommerdijk, N.A.J.M.;

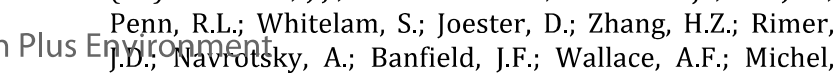


F.M.; Meldrum, F.C.; Cölfen, H.; Dove, P.M. Crystallization by Particle Attachment in Synthetic, Biogenic, and Geologic Environments. Science, 2015, 349, aaa6760, 1-9. 


\section{Anisotropic Time-Resolved Dynamics of Crystal Growth Induced by a Single Laser Pulse Nucleation}

Sarah Al Gharib,1,2 Abdel Karim El Omar, ${ }^{2}$ Adnan $\mathrm{Naja}^{2}$ Ariane Deniset-Besseau, ${ }^{1}$ Sergey A. Denisov, ${ }^{1}$ Pascal Pernot, ${ }^{1}$ Mehran Mostafavi, ${ }^{1}$ Jacqueline Belloni ${ }^{1}$

Université Paris-Saclay, CNRS, Institut de Chimie Physique, 91405, Orsay, France.

Université Libanaise, Laboratoire Physique et Modélisation, 1300 Tripoli, Lebanon

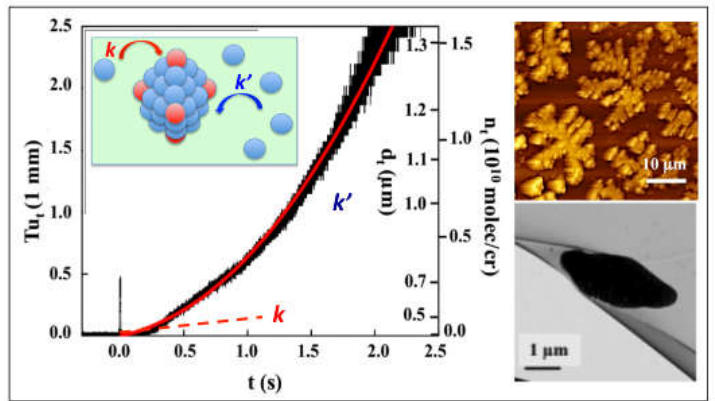

Kinetics signal and mechanism of the turbidity of a $\mathrm{KNO}_{3}$ suspension of crystals induced by a single laser pulse in a supersaturated solution. AFM and TEM images of final crystals. 